\title{
Difference in muscle blood flow fluctuations between dynamic and static thigh muscle contractions: How to evaluate exercise blood flow by Doppler ultrasound
}

\author{
Takuya Osada ${ }^{1,2 *}$ and Göran Rådegran ${ }^{3}$ \\ ${ }^{1}$ Rehabilitation Center, Tokyo Medical University Hospital, Tokyo, Japan \\ ${ }^{2}$ Cardiac Rehabilitation Center, Tokyo Medical University Hospital, Tokyo, Japan \\ ${ }^{3}$ Lund University, Department of Clinical Sciences Lund, Cardiology, Lund, and The Section for Heart Failure and Valvular Disease, VO. Heart and Lung Medicine, \\ Skåne University Hospital, Lund, Sweden
}

\section{Introduction}

Determination of limb blood flow (BF) in relation to exercise may be useful in rehabilitation programs, increasing the general knowledge on oxygen supply, energy metabolism as well as on central and peripheral hemodynamics.

Peak leg muscle oxygen uptake has previously been found to be closely related to the diameter of the feeding artery, which may vary in relation to the dependent muscle mass and oxygen need [1]. The time course of BF alterations may be influenced by remodeling of the arterial structure or restricted motor control as seen in musculoskeletal disorders like disuse syndrome and cerebrovascular disorders with hemiplegia [2-5].

Exercise hyperemia with vasodilation is related to intrinsic (endothelial-related factors, autacoid substances, metabolite and myogenic response) as well as extrinsic (autonomic nerve regulation, signal/reflexes with central command and exercise pressor reflexes with mechanical muscle contraction/accumulated metabolite product) regulation, as well as changes in arteriovenous pressure gradient due to the muscle pump. Furthermore, during exercise the increase in limb oxygen uptake (calculated as the product of exercising arterial BF and the arteriovenous oxygen difference to the exercising limb) is directly proportional to the work performed in relation to the interplay between cardiovascular regulation and muscle energy metabolism. Therefore, determination of limb BF response to exercise through physical training may yield information about an integrated circulatory adaptation and strengthening of muscle force at the target of exercise/rehabilitation prescription. Furthermore, the comparison of hemodynamics during transient exercise such as repeated limb muscle contractions between pre- and post-physical therapy may increase our understanding of the peripheral BF adjustment (so called physical training inducedcirculatory adaptation)

Non-invasive Doppler ultrasound with a high temporal resolution can continuously detect alterations in pulsatile blood velocity profiles as "time and space-averaged and amplitude, signal intensity weighted mean blood velocity" in the conduit artery. The arterial BF can be calculated as the mean blood velocity multiplied by the cross-sectional area in the target artery.

Based on this technique, rapid changes in time courses of blood velocity profiles in the conduit artery have been found, with muscle contraction and/or muscle relaxation during exercise (dynamic/static), in different states of muscle contraction time/frequency and workload, and in relation to vasodilatation/vasoconstriction. Furthermore, the determination of a comprehensive exercise BF, for instance in a brachial, femoral or popliteal artery feeding a limb working muscle group can also be performed during muscle contractions such as with the exercise model of forearm handgrip, lower limb knee extensor or plantar flexion exercise.

Following our previous reports with the series of investigation for muscle/exercise BF regulation during limb exercise using Doppler ultrasound [6-15], large differences have been observed in the time course of the magnitude of the blood velocity profile during steadystate muscle contraction-relaxation phases and between dynamic and static muscle contraction. This raises the issue how to determine exercise BF optimally during repeated muscle contractions.

In general, an optimal/valid BF in a non-exercise limb may exhibit minimum physiological BF variability using samplings of cardiac beatby-beat cycle (BBcycle). However, during muscle contractions, the muscle contraction-induced blood velocity profile in the working limb muscle may be greatly influenced by the magnitude of intramuscular pressure variation and the superimposed influence of perfusion pressure variation. Thus, for determination of optimal exercise BF we must consider how to treat the minimum physiological variability in exercise BF via muscle contraction-relaxation cycle (CRcycle) and/or cardiac BBcycle.

The present commentary visualizes how to determine BF during exercise in relation to CRcycle or BBcycle, in dynamic/isotonic and static/isometric exercise, utilizing the knee extensor model and the Doppler ultrasound technique.

Correspondence to: Takuya Osada, MD, PhD, Rehabilitation Center, Cardiac Rehabilitation Center, Tokyo Medical University Hospital, 6-7-1, Nishishinjuku, Shinjuku-ku, Tokyo 160-0023, Japan, Tel: +81-33342-6111; Fax: +81-333427082; E-mail: dentacmac@aol.com

Key words: muscle blood flow fluctuations, dynamic and static muscle contractions, knee extensor exercise, Doppler ultrasound

Received: December 04, 2016; Accepted: December 21, 2016; Published: December 30, 2016 


\section{One-legged repeated knee extensor exercise model}

Previous studies reported measurement of exercising leg BF by the invasive thermodilution technique, utilizing the one-legged, dynamic (so called isotonic or rhythmic) knee-extensor exercise model $[16,17]$. However, this invasive technique has the limitation of not being able to detect the beat-by-beat blood velocity profile for temporal duration in real time. Whereas many of the available techniques are impaired by different methodological limitations, the indicator thermodilution and non-invasive Doppler ultrasound methods have both been found to give repeatable measurements of the same magnitude during both rest and dynamic knee extensor exercise [18,19]. Furthermore, the thermodilution measurements obtained under similar experimental conditions by Andersen et al. [18] are statistically similar to those obtained by Doppler ultrasound.

In one-legged, dynamic/static knee-extensor exercise, the exercise (absolute workload using the unit watt, $\mathrm{W}$ or relative maximum

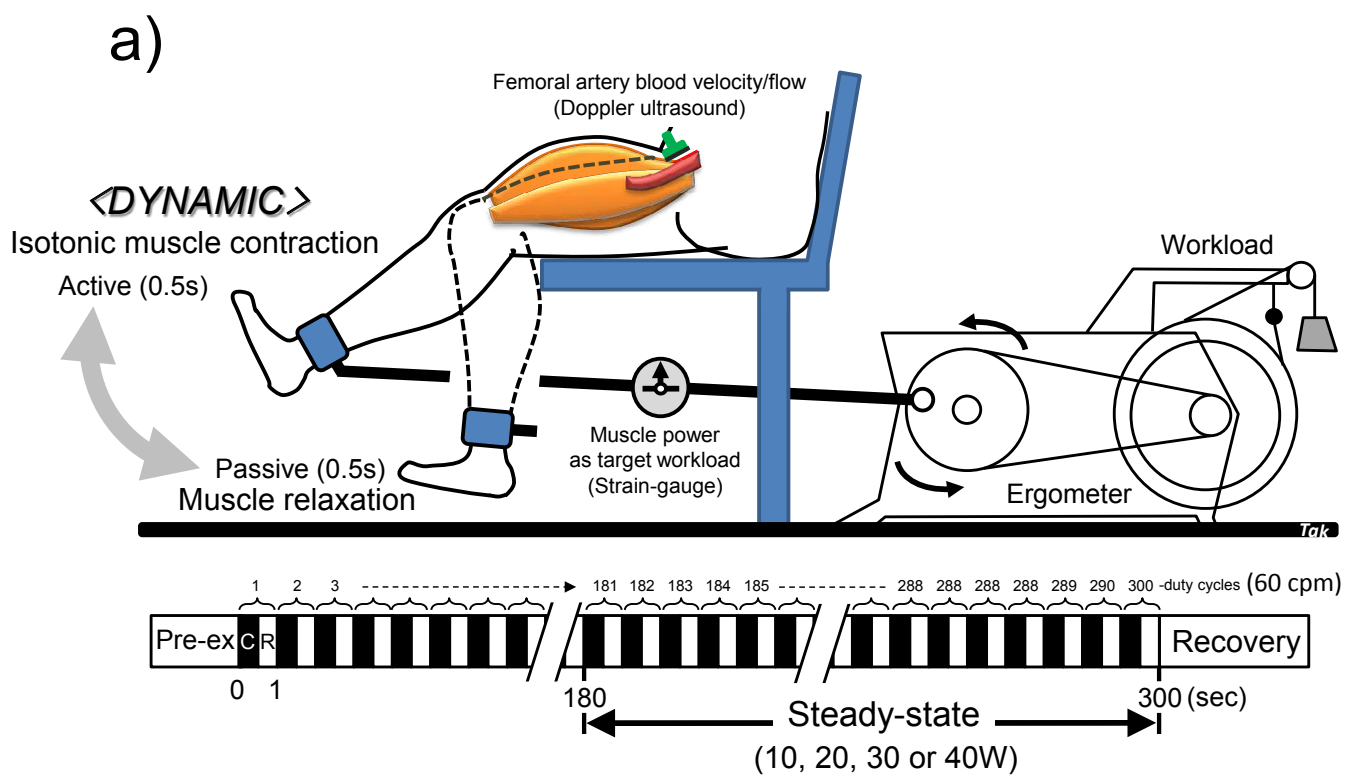

b)

〈STATIC〉

Isometric muscle contraction

Muscle relaxation

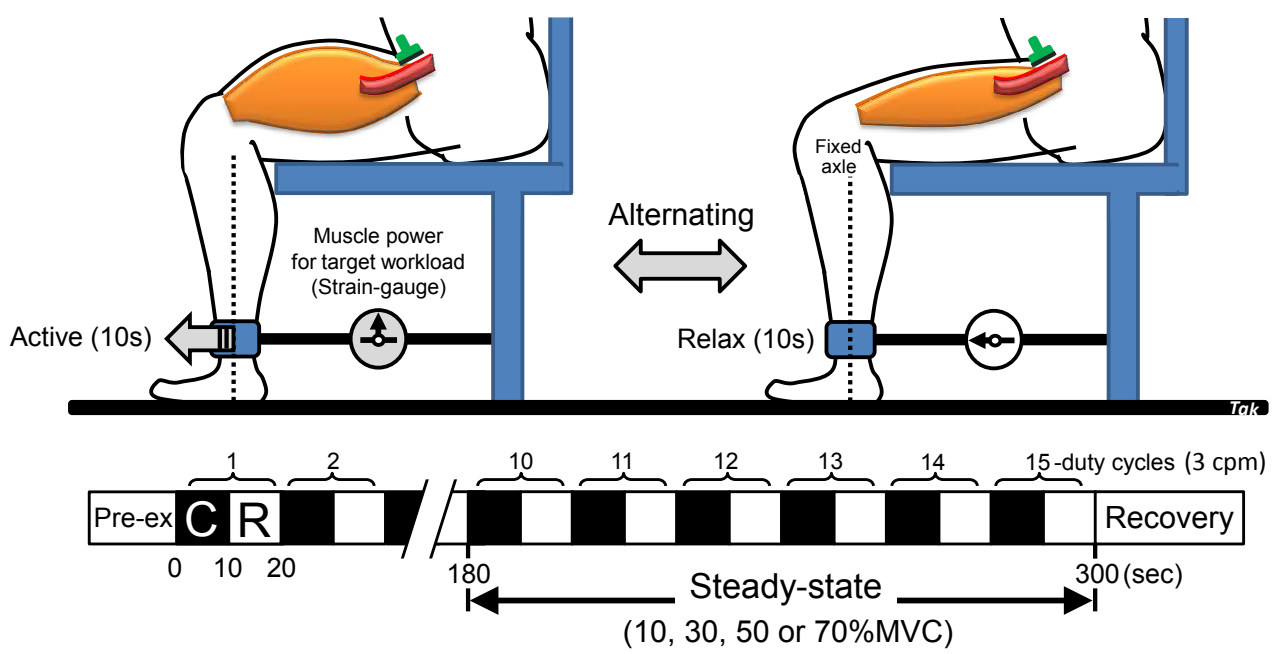

Figure 1. One-legged knee extensor exercise model

a) $<$ Dynamic $>$ Isotonic muscle contraction at 60 contractions per minute performed as $0.5 \mathrm{~s}$-voluntary (active) muscle contraction and $0.5 \mathrm{~s}$-passive muscle relaxation (1s) for 5 min at 10 , 20,30 and $40 \mathrm{~W}$, respectively.

b) $<$ Static $>$ Repeat isometric muscle contraction performed as $10 \mathrm{~s}$-voluntary (active) isometric muscle contraction and $10 \mathrm{~s}-\mathrm{muscle}$ relaxation $(20 \mathrm{~s})$ for 5 min at $10 \%$, $30 \%, 50 \%$ and $70 \% \mathrm{MVC}$, respectively.

The voluntary contraction rhythm was maintained by following the pace of a visible and audible metronome and by visualizing the contraction frequency displayed in real time on a monitor Simultaneous recording of hemodynamic parameters was measured at a steady-state from 3 min to 5 min. Pre-ex, pre-exercise; \%MVC, percentage of maximum voluntary contraction; C, muscle contraction phase; $\mathrm{R}$, muscle relaxation phase. 

ultrasound

voluntary contraction (MVC) using the unit percentage of MVC, $\% \mathrm{MVC}$ ) is confined to the quadriceps muscle group (Figure 1). This model allows stable measurements of femoral arterial blood velocity using Doppler ultrasound, in comparison to treadmill walking or running models, which do not allow BF measurement in the feeding conduit femoral artery, due to the difficulty of insonation $[6,8,10$ $15,18,19]$.

The measurement of blood velocity in the femoral artery feeding the active thigh muscles using Doppler ultrasound, where BF is determined by the product of blood velocity and cross-sectional area, has been validated and shown to produce accurate absolute values both at rest and during incremental leg exercise such as rhythmical/dynamic [18] or static thigh muscle contractions [15]. Thus, the high temporal resolution of the Doppler ultrasound enables continuous measurement of blood velocity throughout the kicking duty cycle during one-legged dynamic/static knee extensor exercise.

The measurement site of the femoral artery was distal to the inguinal ligament, but above the bifurcation into the branches of the superficial and deep femoral arteries. This location minimizes turbulence from the femoral bifurcation and the influence of blood velocity from the inguinal region. Furthermore, the changes of the vessel diameter of the conduit artery in the target location are mostly unaffected by muscle contractions and relaxations $[6,8,15,18]$. Therefore, changes in blood velocity may potentially correspond to changes in $B F$ because $B F$ is calculated by the product of mean blood velocity and the stable crosssectional area in the target artery.

Regarding the hemodynamics in the leg conduit femoral artery during knee extensor exercise, we have previously: 1) validated the method during exercise [18], 2) determined changes in BF due to incremental work intensity at different muscle contraction frequencies $[8,15]$, and evaluated 3 ) the physiological variability/fluctuations in the magnitude of blood velocity/BF due to the muscle contraction and relaxation phases [10-13], as well as 4) changes in BF due to spontaneous changes of workload at a certain target intensity and muscle contraction per minute (cpm) rate [14]. The exercise model presented in this commentary was 1) dynamic muscle contractions at $60 \mathrm{cpm}$ (0.5s-on and 0.5s-off) at 10, 20,30 and $40 \mathrm{~W}$ (Figure 1a), 2) repeated static muscle contractions at $3 \mathrm{cpm}$ (10s-on and 10s-off) at $10 \%, 30 \%, 50 \%$ and $70 \%$ of MVC (Figure $1 \mathrm{~b}$ ).

\section{Blood velocity/flow fluctuations due to voluntary mus- cle contraction and cardiac contractions}

The difference between dynamic and static muscle contractions depends on the time period of muscle contraction and relaxation phases (corresponding to muscle contraction frequency). We therefore focused on the magnitude of blood velocity/BF between muscle contraction and relaxation phases, and its variations between CRcycle and $\mathrm{BBcycle}$ using dynamic $(60 \mathrm{cpm})$ and static $(3 \mathrm{cpm})$ exercise.

As shown in Figure 2, continuous recordings of hemodynamic parameters can demonstrate the magnitude of the physiological variability in blood velocity during dynamic- and repeated static-thigh muscle contractions.

In dynamic/isotonic exercise at $60 \mathrm{cpm}(0.5 \mathrm{~s}$-on and $0.5 \mathrm{~s}$-off), the continuous blood velocity curve during repeated muscle contractions fluctuated rapidly due to the muscle force curve, which indicated that the $0.5 \mathrm{~s}$-muscle contraction restricted BF (temporal reduced blood velocity), and consequently $0.5 \mathrm{~s}$-muscle relaxation may induce an increase in BF (higher blood velocity) (Figure 2a). Theoretically, the rate of $60 \mathrm{cpm}$ (single muscle contraction-relaxation cycle in 1s) may be able to uniformly interfere with the magnitude of the single whole blood velocity profile (interval $<1 \mathrm{~s}$ ) when the heart rate is above 60 beats $/ \mathrm{min}$ (i.e. time interval of muscle contraction similar to heart rate) during exercise. This means that rapid changes of muscle force curve every second may completely disturb the formal blood velocity profile (time and space-averaged and amplitude as the clear systolic and diastoliclike profile, blood pressure curve-like). Therefore, we acknowledge the muscle contraction-induced physiological BF variability with systole and diastole superimposed by CRcycle.

Figure 3a (corresponding to an expanded view indicated in Figure 2a) clearly shows the four specific variations with magnitude of the physiological variability in blood velocity with muscle contraction or relaxation-induced variations in muscle force, and consequently the intramuscular pressure variations, along with the additional influence of blood pressure, as well as the tonic influence of the state of vasodilatation. An oscillation in the peak of the blood velocity is partially related to the interaction between peak muscle force strength and the peak blood pressure curve. In particular, the magnitude of the tip point of blood velocity profile for a single muscle contraction or muscle relaxation with single systolic or diastolic phase was found to be closely related to the point in a peak systolic- or second peak diastolicblood pressure curve throughout the muscle contraction and muscle relaxation phases as determined by muscle force curve and/or the electromyography signals.

There is a relatively high $\mathrm{BF}$ component at the systolic phase during muscle relaxation (RS), and at the diastolic phase during muscle relaxation (RD), as compared to the relatively low (reduced) $\mathrm{BF}$ component at the systolic phase during muscle contraction (CS) and the diastolic phase during muscle contraction (CD) in Figure 3b. Furthermore, BF during both systolic and diastolic phases during the muscle relaxation phase showed a positive linear increase with workload. However, during the muscle contraction phase it was similar between workloads [11].

Regarding the variability in $\mathrm{BF}$ during dynamic exercise, discrepancies in variability of BF between BBcycle and CRcycle are seen at $30 \mathrm{~W}$ and $40 \mathrm{~W}$ (Figure $4 \mathrm{a}$ ). These higher variations in $\mathrm{BF}$ during the $\mathrm{BBcycle}$ may be due to the large increase in $\mathrm{BF}$ in the systolic or diastolic phase during muscle relaxation compared to muscle contraction at 30 $\mathrm{W}$ and $40 \mathrm{~W}$. However, the variability in BF evaluated by CRcycle is similar (almost $15 \%$ of coefficients of variation) between workloads. Therefore, the approach for determination of optimal steady-state $\mathrm{BF}$, including the samplings for CRcycle $(\bullet$ in Figure $2 \mathrm{a})$ is better, even if the measurement of $\mathrm{BF}$ at rest is generally valid using $\mathrm{BBcycle}$ samplings ( $\boldsymbol{\Delta}$ in Figure 2a).

In static/isometric exercise at $3 \mathrm{cpm}$ (10s-on and 10s-off) described in Figure 2b, the blood velocity profile clearly presents a clear systolic and diastolic-like profile (blood pressure curve-like). This is due to non-oscillation of the blood velocity profile (a non-disturbed blood velocity profile), lacking rhythmical vessel compression even under 10s-sustained isometric muscle contraction. Any mechanical compression remains extravascular with increasing intramuscular pressure. Therefore, the variations in beat-by-beat blood velocity are similar between \%MVC (approximately 15\%, range: $12.4-17.8 \%$ ) and show no relation to muscle contraction intensity (Figure $4 \mathrm{~b}$ ). This finding supports changes in beat-by-beat blood velocity being lower during 10s-sustained isometric muscle contraction, although a gradual 

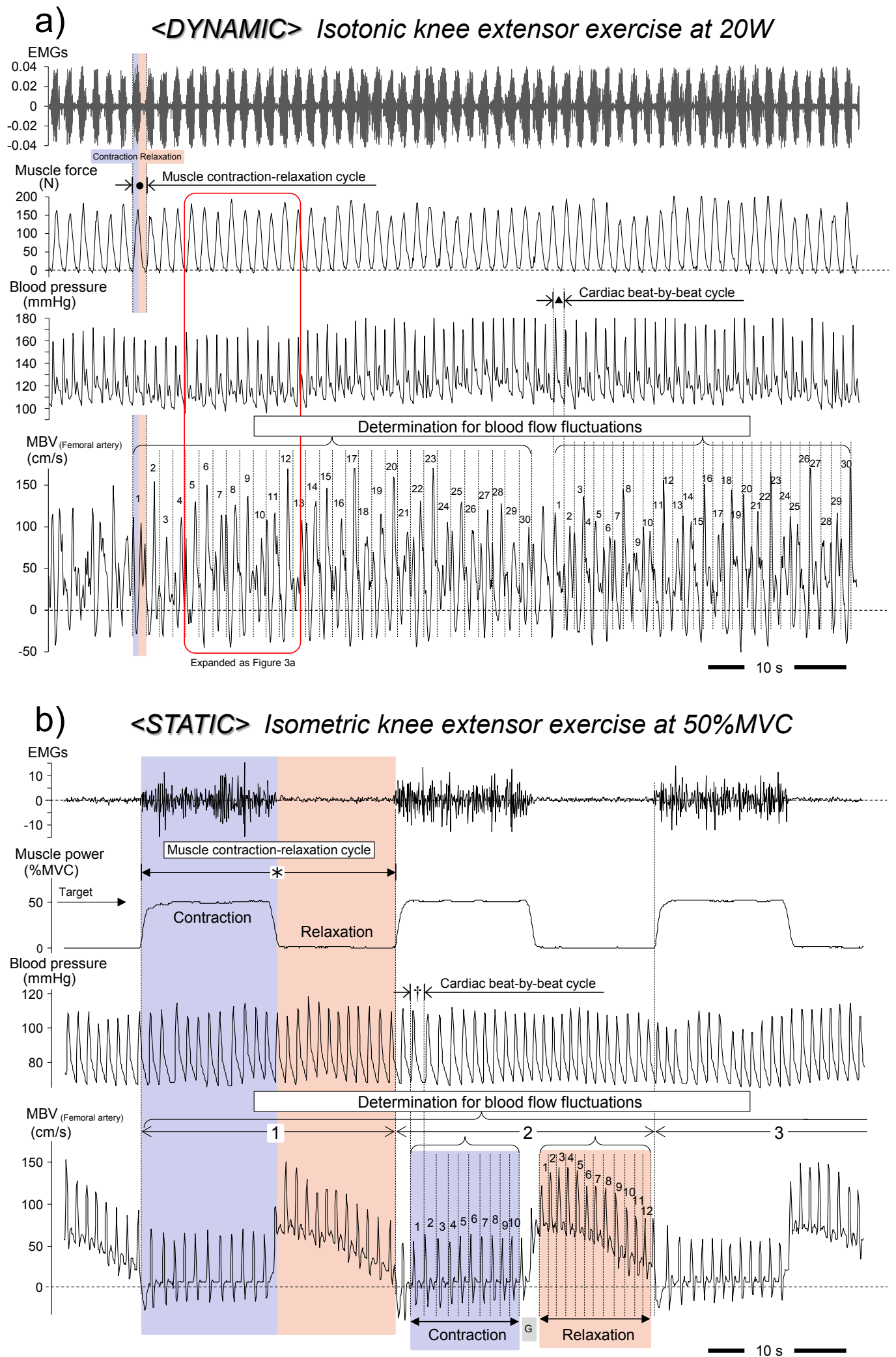

Figure 2. Simultaneous recording of blood velocity and hemodynamic parameters during exercise

a) $<$ Dynamic $>$ Isometric muscle contractions were clearly evident in the oscillations of mean blood velocity (MBV) due to muscle contraction-relaxation cycle superimposed on the cardiac beat cycle. The MBV profile shows turbulence representing non-systole and diastole portions. b) $<$ Static $>$ Repeat isometric muscle contraction may indicate the clear MBV profile at each beat corresponding to the cardiac systole-diastole. The blood velocity fluctuations (coefficients of variation) were determined by each muscle contraction-relaxation cycle $(\bullet$ or $*$ ) as well as the cardiac beat-by-beat cycle ( $\boldsymbol{\Lambda}$ or $\dagger$ ). EMGs, surface electromyography; $\mathrm{G}$, gap between contraction and relaxation. Figure $2 \mathrm{a}$ is drawn from our original unpublished data. Figure $2 \mathrm{~b}$ is adapted from Osada et al. [15], reproduced with permission for unrestricted use from BioMed Central. 
a)

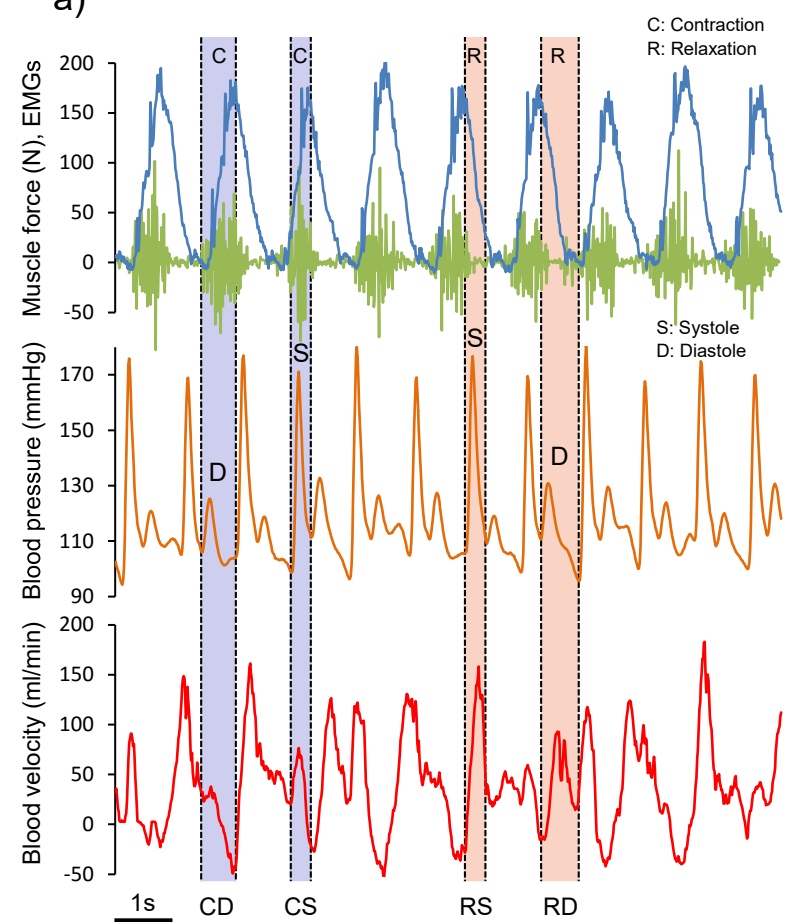

b)

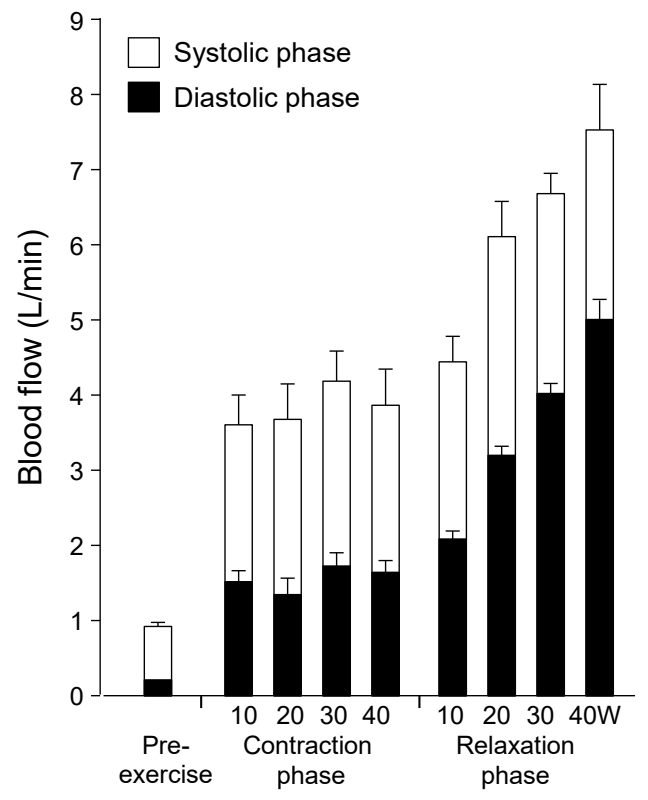

Figure 3. Alterations in blood velocity profile during muscle contractions-relaxation superimposed on cardiac cycle

a) A partial window in the simultaneous recording in Figure 2a was expanded to show the influence on the blood velocity profile by muscle force curve, EMGs (muscle contractions) and blood pressure curve (cardiac beat). Temporal blood velocity profile curves were closely related to the blood pressure curve and muscle force curve. The magnitude of the peak of blood velocity profiles for a single muscle contraction or muscle relaxation with single systolic or diastolic phase was found to be closely related to the peak systolic or second peak diastolic blood pressure curve through muscle contraction-relaxation phases. Muscle contraction synchronized systole (CS) or diastole (CD); Muscle relaxation synchronized systole (RS) and diastole (RD); EMGs, surface electromyography.

b) Blood flow was different between muscle contraction and muscle relaxation phases, consecutively provided in systolic and diastolic phase. Furthermore, blood flow during muscle relaxation synchronized with systolic and diastolic phase showed a positive linear correlation with work rate. The values are expressed as means \pm standard error.

Figure 3a is drawn from our original unpublished data. Figure 3b is adapted from Osada and Rådegran [13], reproduced with permission from The Physiological Society of Japan.

a)

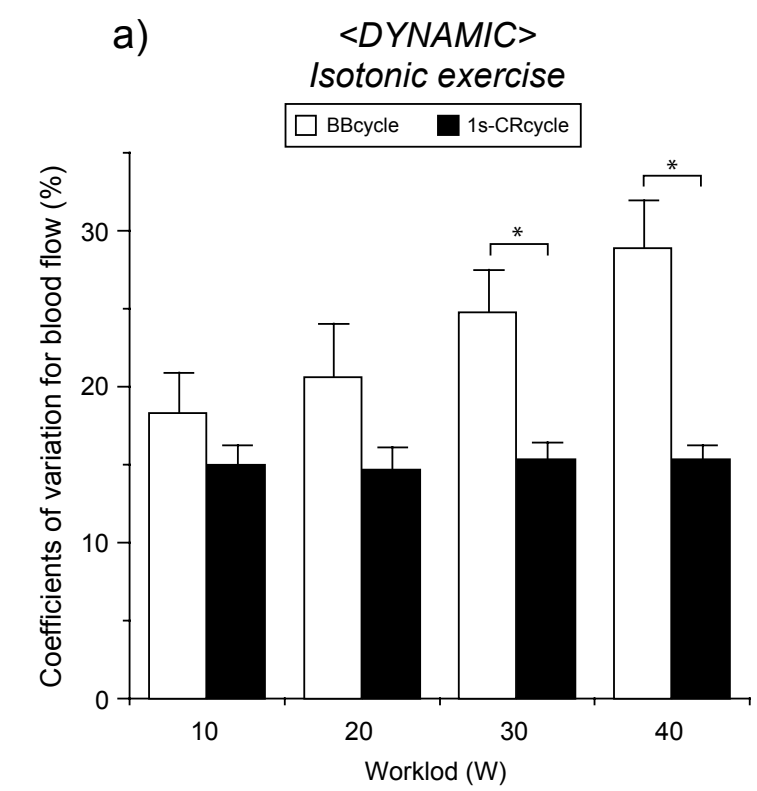

b)
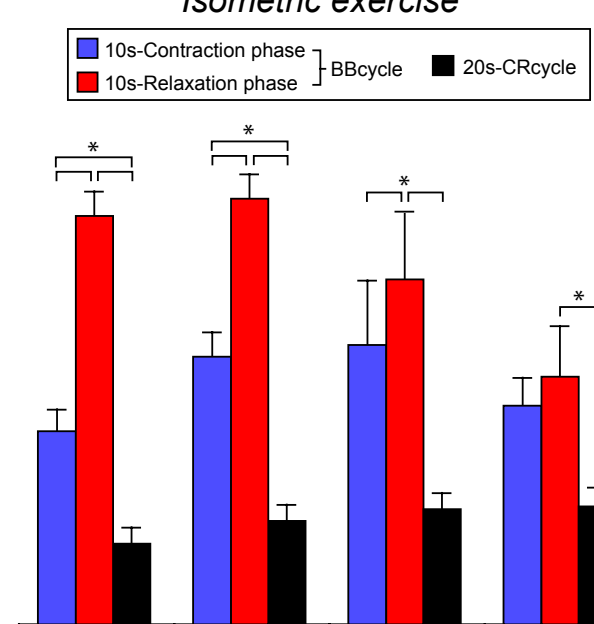

10
$<S T A T I C>$

Isometric exercise

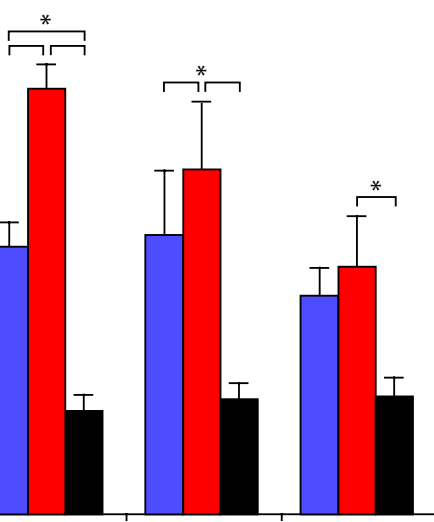

30

70

$\% \mathrm{MVC}$

Figure 4. Blood flow fluctuations (variations) determined by muscle contraction or cardiac contraction cycle during dynamic and static exercise

a) $<$ Dynamic $>$ isotonic exercise; Significantly higher blood flow variability (coefficients of variations) was determined by the cardiac beat-by-beat cycle (1-BBcycle) than muscle contractionrelaxation cycle (1-CRcycle, $1 \mathrm{~s}$ ) at $30 \mathrm{~W}$ and $40 \mathrm{~W}$, although blood flow variability was similar at each workload when determined for 1-CRcycle.

b) $<$ Static $>$ isometric exercise; There was a significant difference in the blood flow variability between 1-BBcycle during 10s-muscle contraction phase or 10s-muscle relaxation phase and $1-\mathrm{CRcycle}(20 \mathrm{~s})$ at each percentage of maximum voluntary contraction $(\% \mathrm{MVC})$. *Significant difference $(\mathrm{p}<0.05)$ - one way ANOVA. The values are expressed as means \pm standard error. Figure 4a adapted from Osada and Rådegran [12], reproduced with permission from Edizioni Minerva Medica. Figure $4 \mathrm{~b}$ is our original unpublished data newly analyzed by the source in Ref. [15]. 
a)

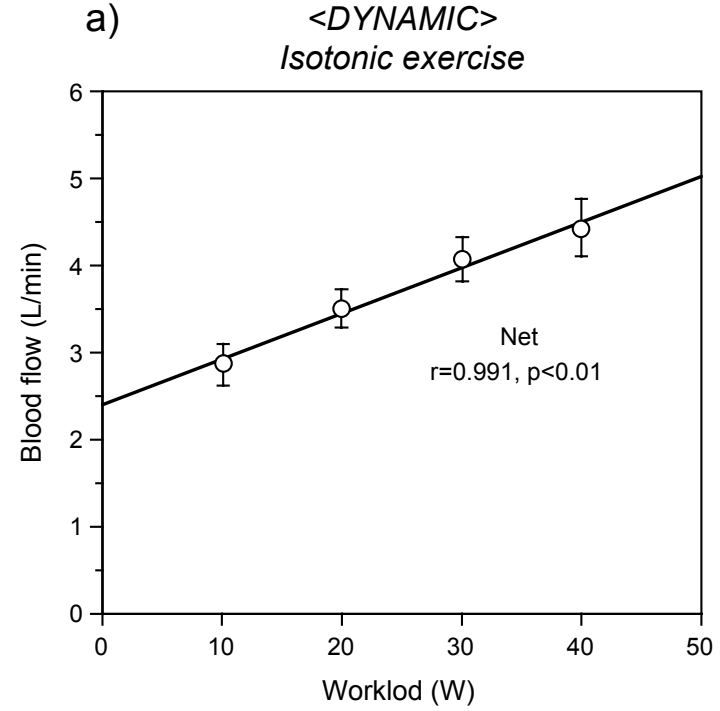

b)

$<S T A T I C>$

Isometric exercise

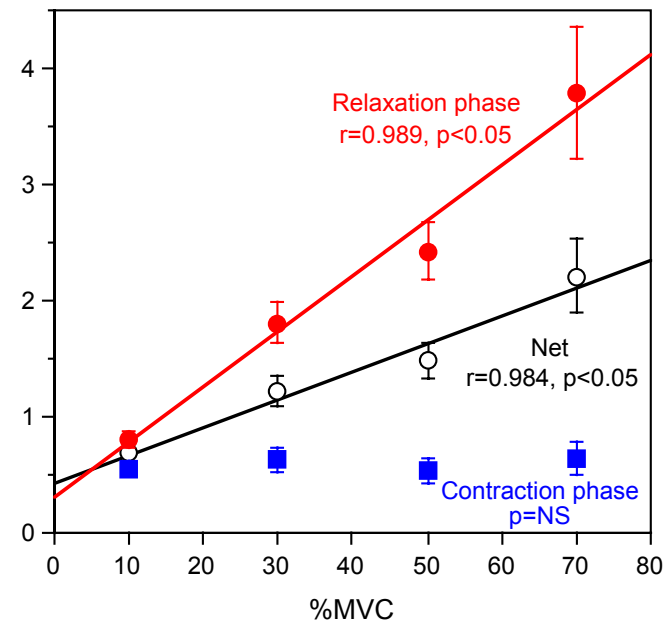

Figure 5. Relationship between blood flow and exercise intensity

a) $<$ Dynamic $>$ There was a close positive linear relationship between net-blood flow and workload $(r=0.991, p<0.01)$. b) $<$ Static $>$ There was a close positive linear relationship between blood flow and \%MVC during muscle relaxation $(r=0.989, \mathrm{p}<0.05)$ as well as combined muscle contraction-relaxation $(\mathrm{r}=0.984, \mathrm{p}<0.05)$. However, blood flow during isometric muscle contraction showed no change within the target \%MVC. Statics $(\mathrm{p}<0.05$ and correlation coefficient, $\mathrm{r})$ - linear regression analysis. NS, not significant; \%MVC, percentage of maximum voluntary contraction. The values are expressed as means \pm standard error. Figure 5a adapted from Osada and Rådegran [12], reproduced with permission from Edizioni Minerva Medica. Figure $5 \mathrm{~b}$ adapted from Osada et al. [15], reproduced with permission for unrestricted use from BioMed Central.

but not significant BF increase was seen during 10s-sustained isometric muscle contraction [15]. It means that during 10s-sustained isometric muscle contraction the BF measured by a few beat-by-beat samplings may be equal to the essential $\mathrm{BF}$ value during the muscle contraction phase.

In contrast, the variations in beat-by-beat BF evaluated by BBcycle were significantly higher in 10 s-muscle relaxation than in 10 s-isometric muscle contraction at 10\%MVC-50\%MVC (Figure 4b). However, the low value for beat-by-beat $\mathrm{BF}$ variation at $70 \% \mathrm{MVC}$ suggests that the higher blood velocity (prolonged vasodilation) was maintained during the 10s-muscle relaxation phase. This result suggests that during 10s-muscle relaxation the time course of the beat-by-beat $\mathrm{BF}$ magnitude may markedly change (high attenuation ratio for beat-bybeat $\mathrm{BF}$ from onset to end of $10 \mathrm{~s}$-muscle relaxation) below $50 \% \mathrm{MVC}$, even if the major variation of the blood velocity consequently occurs during the muscle relaxation phase.

The magnitude of BF at $10 \% \mathrm{MVC}-50 \% \mathrm{MVC}$ and the difference in $\mathrm{BF}$ variations between 10s-isometric muscle contraction and relaxation may indicate the characteristic of exercise hyperemia (describing the exponential decay of beat-to-beat BF magnitude) after the end of 10 s-muscle contraction during repeated isometric muscle contraction (see the blood velocity magnitude in contraction and relaxation phase in Figure 2b) [15]. As mentioned above, we must recognize that there is a large difference in single blood velocity value between first and last beats during the muscle relaxation phase, and the variations in beat-bybeat $\mathrm{BF}$ are obviously high below $50 \% \mathrm{MVC}$.

The message from these findings is that if a shorter duration of beat-by-beat samplings during muscle relaxation were used for $\mathrm{BF}$ determination, the resulting $\mathrm{BF}$ value would potentially be an overor under-estimation. We must remain aware that BF measured by transient beat-by-beat sampling may not be expressing the essential BF value during muscle relaxation phase.

As expected, variations in BF measured by CRcycle $\left({ }^{*}\right.$ in Figure 2b) were significant lower by BBcycle ( $\dagger$ in Figure $2 \mathrm{~b}$ ) during 10 s-isometrcic muscle contraction (10\%MVC and 30\%MVC), and during muscle relaxation (10\%MVC-70\%MVC). This demonstrated that the comprehensive (net-) $\mathrm{BF}$ including the muscle contraction and relaxation phase (i.e. CRcycle) will naturally be less variable in comparison to the relatively high $\mathrm{BF}$ variations by $\mathrm{BBcycle}$ during muscle contraction and/or relaxation. The optimal determination of steady-state BF might require CRcycle sampling for repeated isometric muscle exercise.

\section{Speculated mechanisms for controlling BF during exer- cise in relation to CRcycle}

The cardiovascular responses to exercise are coordinated by signals from central motor systems and peripheral sensors such as baroreceptors, muscle chemocensors and mechanoreceptors being integrated by the central nervous system with the degree of exercise/ muscle contraction intensity. The central nervous system may subsequently influence heart rate, cardiac output and vascular tone, and thus skeletal muscle BF by altering sympathetic and parasympathetic nervous activity. Furthermore, exercise-induced muscle hyperemia has consequently been controlled by an inter-play between both "feed back" and "feed forward" vascular control mechanisms which include central mechanisms (neural and hormonal factors), as well as local mechanisms involving the myogenic activity, and mediators derived from the endothelium, muscle fibers and/or muscle mechanical factors.

In the knee extensor exercise model utilizing a relatively small thigh muscle mass, the local factors regulating BF are thought to be of most importance including the voluntary repeated muscle contractions. It has previously been discussed that muscle contraction-induced rapid alterations in the conduit arterial blood velocity profile may be closely related to the magnitude of intramuscular pressure variation (muscle mechanical factors) and superimposed influence of perfusion pressure variation (pulsatile hemodynamic factors) [10,11,13-15,20-22]. During steady-state, high intramuscular pressure during muscle contractions 

ultrasound

may consequently temporarily reduce or even reverse the blood velocity, depending on the relationship between the intramuscular pressure and arterial blood pressure. The major extent of the blood velocity and flow consequently occurs during the muscle relaxation phase.

Finally, the time and space-averaged blood velocity/flow magnitude/dynamics may represent the phenomenon with blood velocity changes (changes in vasodilation) due to CRcycle by above mentioned integrated cardiovascular adjustment. The high time resolution blood velocity profile in continuous recording may be suitable for the investigation of $\mathrm{BF}$ regulation (time course in rapid changes in blood velocity profile described in Figure 2).

\section{Exercising blood flow versus exercise intensity}

Limb conduit artery BF in the working muscle is one indicator of metabolic demand in a local large muscle group. Therefore, exercising net-BF increased positively and linearly with increasing target intensity (dynamic exercise as workload and static exercise as \%MVC) as shown in Figure 5. Interestingly, regarding static exercise, there was a close positive linear relationship between net-BF and \%MVC, and between $\mathrm{BF}$ during muscle relaxation phase and \%MVC, but not during the isometric muscle contraction phase. The evaluation of the magnitude for blood velocity profiling was possible using Doppler ultrasound with high temporal resolution. The important message from our findings is that accounting for the time resolution changes in the blood velocity due to, i.e., CRcycle and/or BBcycle is valuable information for the measurement of target exercise $\mathrm{BF}$.

\section{Acknowledgements}

The authors acknowledge the long-term support of professor emeritus Bengt Saltin, the staff of the Copenhagen Muscle Research Centre, and the volunteers who participated in the studies. The study was supported by the Danish National Research Foundation Grant 50414, as well as the "Excellent Young Researchers Overseas Visit Program" in Scientific Research (No. 21-8285) 2010 and Scientific Research (C) general (No. 15K01730) from MEXT and JSPS (T. Osada).

\section{Authorship contributions}

T. Osada contributed to the conception and design of the study, data acquisition, analysis and interpretation. T. Osada and G. Rådegran were involved in drafting, revising and finalization of the manuscript.

\section{Conflicts of interest}

The authors declare no conflicts of interests in relation to the article.

\section{References}

1. Rådegran G, Saltin B (2000) Human femoral artery diameter in relation to knee extensor muscle mass, peak blood flow, and oxygen uptake. Am J Physiol Heart Circ Physiol 278: H162-167. [Crossref]

2. Ivey FM, Gardner AW, Dobrovolny CL, Macko RF (2004) Unilateral impairment of leg blood flow in chronic stroke patients. Cerebrovasc Dis 18: 283-289. [Crossref]
3. Billinger SA, Kluding PM (2009) Use of Doppler ultrasound to assess femoral artery adaptations in the hemiparetic limb in people with stroke. Cerebrovasc Dis 27: 552 558. [Crossref]

4. Ivey FM, Hafer-Macko CE, Ryan AS, Macko RF (2010) Impaired leg vasodilatory function after stroke: adaptations with treadmill exercise training. Stroke 41: 2913 2917. [Crossref]

5. Durand MJ, Murphy SA, Schaefer KK, Hunter SK, et al. (2015) Impaired hyperemic response to exercise post stroke. PLoS One 10: e0144023. [Crossref]

6. Osada T, Katsumura T, Hamaoka T, Inoue S, Esaki K, et al. (1999) Reduced blood flow in abdominal viscera measured by Doppler ultrasound during one-legged knee extension. J Appl Physiol 86: 709-719. [Crossref]

7. Osada T, Katsumura T, Hamaoka T, Murase N, Naka M, et al. (2002) Quantitative effects of respiration on venous return during single knee extension-flexion. Int J Sports Med 23: 183-190. [Crossref]

8. Osada T, Rådegran G (2002) Femoral artery inflow in relation to external and total work rate at different knee extensor contraction rates. J Appl Physiol 92: 1325-1330. [Crossref]

9. Osada T, Katsumura T, Murase N, Sako T, Higuchi H, et al. (2003) Post-exercise hyperemia after ischemic and non-ischemic isometric handgrip exercise. $J$ Physiol Anthropol Appl Human Sci 22: 299-309. [Crossref]

10. Osada T (2004) Muscle contraction-induced limb blood flow variability during dynamic knee extensor. Med Sci Sports Exerc 36: 1149-1158. [Crossref]

11. Osada T, Rådegran G (2005) Alterations in the rheological flow profile in conduit femoral artery during rhythmic thigh muscle contractions in humans. Jpn J Physiol 55: 19-28. [Crossref]

12. Osada T, Rådegran G (2006) Differences in exercising limb blood flow variability between cardiac and muscle contraction cycle related analysis during dynamic knee extensor. J Sports Med Phys Fitness 46: 590-597. [Crossref]

13. Osada T, Rådegran G (2006) Alterations in the blood velocity profile influence the blood flow response during muscle contractions and relaxations. J Physiol Sci 56: $195-$ 203. [Crossref]

14. Osada T, Rådegran G (2009) Femoral artery blood flow and its relationship to spontaneous fluctuations in rhythmic thigh muscle workload. Clin Physiol Funct Imaging 29: 277-292. [Crossref]

15. Osada T, Mortensen SP, Rådegran G (2015) Mechanical compression during repeated sustained isometric muscle contractions and hyperemic recovery in healthy young males. J Physiol Anthropol 34: 36. [Crossref]

16. Andersen P, Saltin B (1985) Maximal perfusion of skeletal muscle in man. J Physiol 366: 233-249. [Crossref]

17. Andersen P, Adams RP, Sjøgaard G, Thorboe A, Saltin B (1985) Dynamic knee extension as model for study of isolated exercising muscle in humans. $J$ Appl Physiol 59: 1647-1653. [Crossref]

18. Rådegran G (1997) Ultrasound Doppler estimates of femoral artery blood flow during dynamic knee extensor exercise in humans. J Appl Physiol 83: 1383-1388.[Crossref]

19. Rådegran G, Saltin B (1998) Muscle blood flow at onset of dynamic exercise in humans. Am J Physiol 274: H314-322. [Crossref]

20. Osada T, Saltin B, Mortensen SP, Rådegran G (2012) Measurement of the exercising blood flow during rhythmical muscle contractions assessed by Doppler ultrasound: Methodological considerations. J Biomed Sci Eng 5: 779-788.

21. Osada T, Saltin B, Rådegran G (2013) Assessment of voluntary rhythmic muscle contraction-induced exercising blood flow variability measured by Doppler ultrasound. Open J Mol Integr Physiol 3: 158-165.

22. Osada T, Murase N, Kime R, Katsumura T, Rådegran G (2014) Blood flow dynamics in the limb conduit artery during dynamic knee extensor exercise assessed by continuous Doppler ultrasound measurements. J Phys Fitness Sports Med 3: 409-421.

Copyright: (C2016 Osada T. This is an open-access article distributed under the terms of the Creative Commons Attribution License, which permits unrestricted use, distribution, and reproduction in any medium, provided the original author and source are credited. 